\title{
Idiopathic Congenital Lymphedema - A Case Report
}

\author{
MD. ABDUL BAKI ${ }^{1}$, SURAIYA BEGUM ${ }^{2}$, AFROZA HAQUE ${ }^{1}$, TAHMINA BEGUM ${ }^{3}$, BEDOWRA ZABEEN ${ }^{1}$, \\ NAZMUN NAHAR ${ }^{3}$
}

\section{Introduction:}

Lymphoedema is defined as regional accumulation of excessive interstitial protein-rich fluid caused primarily by an imbalance between the inflow and the removal of interstitial fluid and protein, and secondarily by a malformation or malfunction of the lymphatic system ${ }^{1}$. There are two major categories of lymphoedema: primary and secondary ${ }^{2}$. Primary lymphedema is a rare disease that results from impaired lymphatic drainage. It is estimated to occur in about 1.15 in 100,000 persons who are less than 20 years old with a sex ratio of about one male to three females ${ }^{3}$. Causes of primary lymphedema are due to congenital absence, hypoplasia or underdevelopment of the superficial lymphatic channel. The disease is limited to the leg but arms, hand and feet can also be affected $^{4}$. If lymphatics are damaged or blocked by any pathologic process like infection, surgical excision, neoplasm, irradiation, or trauma, the interstitial fluid builds up in the tissues causing secondary lymphedema ${ }^{5}$.

Three form of primary lymphedema have been described: (1) lymphedema tarda, with onset in the late second or third decade of life; (2) lymphedema praecox with onset at or near puberty; and (3) congenital lymphedema, noticed at birth or soon thereafter ${ }^{3,4}$. Congenital lymphedema is the rarest form of primary lymphedema, accounting for approximately $1: 60,000$ live births ${ }^{1}$. Congenital lymphedema can be classified into familial (hereditary) and idiopathic (non hereditary) subgroups. When congenital lymphedema is of the hereditary form the eponym Milroy disease is applied. Milroy disease is an autosomal dominant inherited primary congenital lymphedema 6 . Gene has been mapped to the $5 q 35.3$ region with code for vascular endothelial growth factor receptor 3 (VEGFR-3) 6 . Familial primary congenital lymphedema has also been associated with several genetic syndromes including noonan's syndrome,

1. Registrar, Department of Paediatrics, BIRDEM Hospital

2. Assistant professor of Paediatrics, Bangabandhu Sheikh Mujib Medical University, Dhaka

3. Professor of Paediatrics, BIRDEM Hospital

Correspondence: Dr. Md Abdul Baki, Registrar, Department of Paediatrics, BIRDEM Hospital (Cell: 01819116047 email: farhanbaki@gmail.com) distichiasis-lymphedema syndrome, a agenaes' syndrome, yellow nail syndrome, and turner's syndrome ${ }^{7}$. When lymphedema without any dysmorphic features and no family history of lymphedema the eponym idiopathic congenital lymphedema is utilized ${ }^{4}$. Etiology of idiopathic primary congenital lymphedema is unknown and a de novo genetic event of genes involved in lymphangiogenesis is a possibility ${ }^{4}$.

\section{Case report:}

A 15 month old boy presented with swelling of left foot, leg and right hand since 5 days of his age. Initially the swelling was confined to the foot and then it was gradually progressing with involvement of his left foot and leg which was grossly edematous. His hand swelling was restricted up to wrist joint and did not progress further. He also had chronic dermatitis of the face, arms and legs. He had no history of lymphangitis or cellulitis and had no complaint of any pain during walking and standing. He is the only child of nonconsanguineous parents. There was no known family history of limb swelling. He was delivered normally at home without any complications. His birth weight was 3,100 grams. He had a normal growth and development. His physical examination revealed non-pitting edema of left foot, left leg and right hand (Fig.-1). The circumferences in different regions on the left foot and leg were 2 to $6 \mathrm{~cm}$ greater than corresponding regions on the right (Fig.-2). He had no dysmorphic features. There was no localized overgrowth of bone and no varicose veins. His nails were normal. Multiple localized excoriation marks due to dermatitis were found over the face, arms and legs; He had no swelling of the scrotum and penis. There was no evidence of infection. Biochemical tests were done to exclude hepatic or renal etiologies and urinalysis to exclude proteinuria. All of these revealed normal finding. Doppler ultrasonography of affected limbs as well as pelvic and abdominal ultrasonography was done to exclude deep vein thrombosis and obstructive lesions that may present with unilateral limb swelling and was found normal. CFT for filaria was negative. The diagnosis of idiopathic primary congenital lymphedema was confirmed by isotopic lymphatic scintigraphy. Scintigraphy findings revealed 


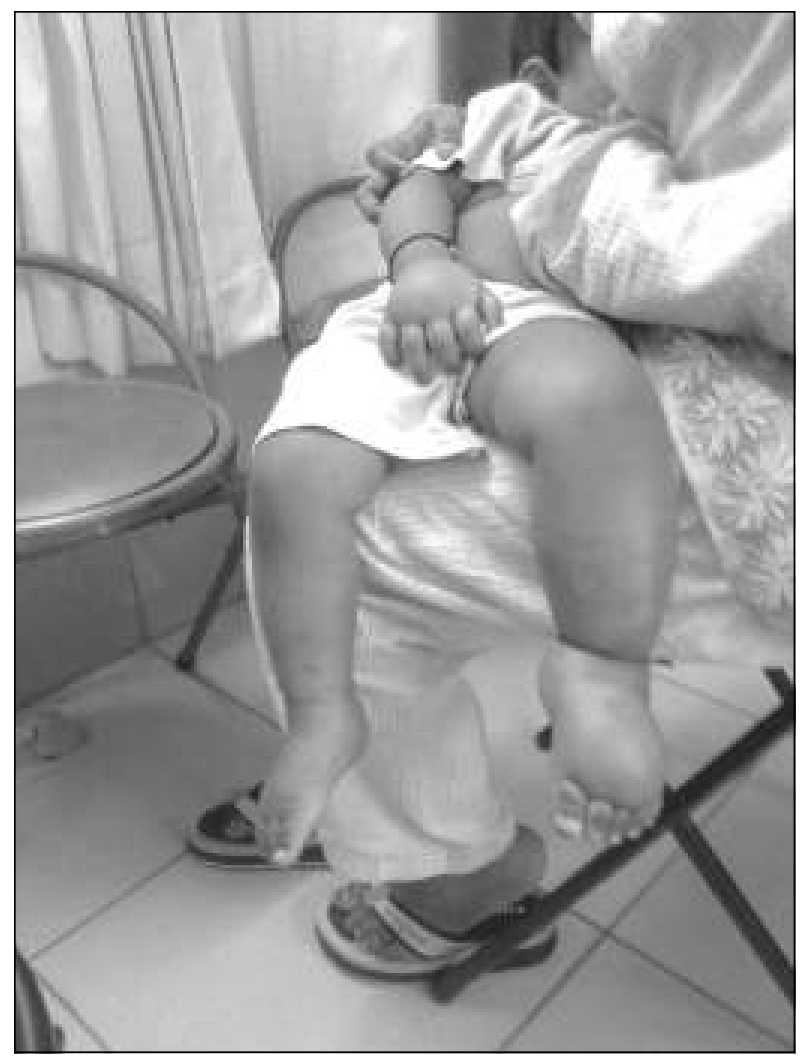

Fig.-1: Swelling of left foot, left leg and right hand

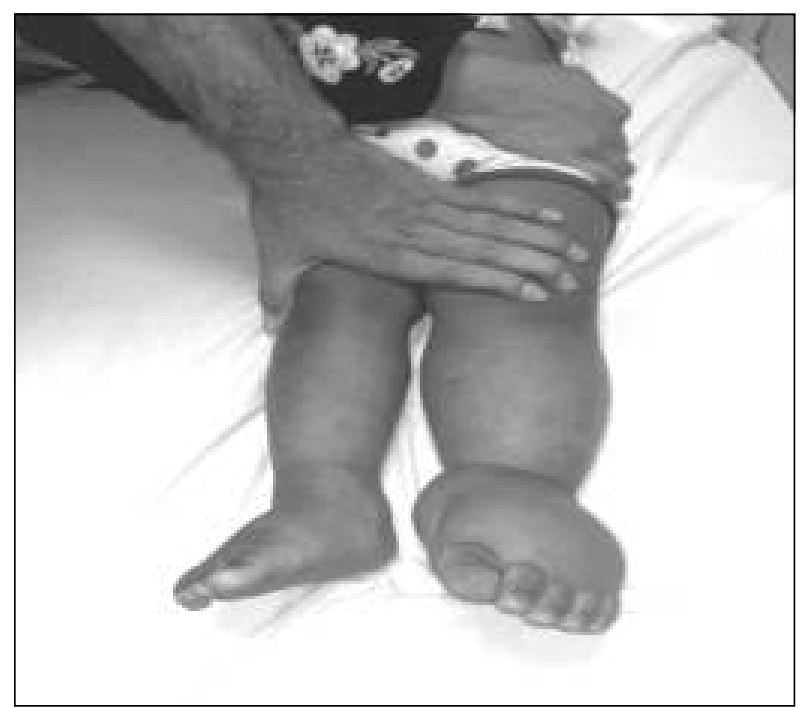

Fig.-2: Unilateral swelling of lower limb

non visualized lymphatic channel and lymph nodes in left lower limb and there was no ascent of tracer into the lymphatic vessels of the left lower extremity (Fig.-3). He was treated conservatively like elevation of affected limbs, skin care and avoiding prolonged standing. Surgical opinion was also taken and adviced to follow up the patient. On follow-up, the swelling did not progress further in next 12 months.

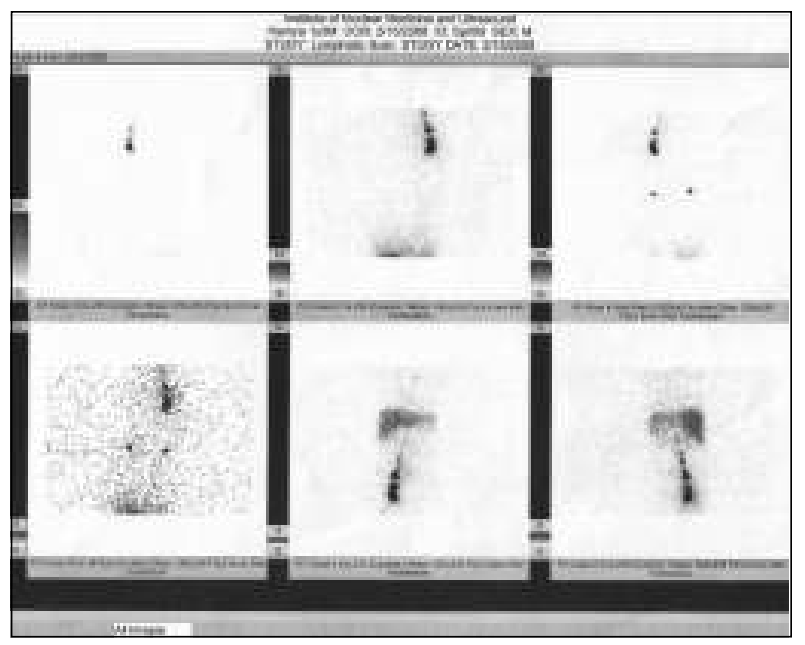

Fig.-3: Lymphoscintigraphy: Non visualized lymphatic channel and lymph nodes in left lower limb

\section{Discussion:}

Idiopathic primary lymphedema is a rare disorder causing persistent swelling in an extremity due to impaired lymphatic drainage. Wananukul reported a case of idiopathic primary congenital lymphedema involving lower limbs and genitalia but patient had no family history of lymphedema and also had no dysmorphic feature ${ }^{5}$. Sim also reported a case of lymphedema without any family history and dysmorphic features and he categorized the case as an idiopathic congenital lymphedema ${ }^{8}$. Our patient also had no family history of lymphedema and no dysmorphic features so we categorized this patient as idiopathic primary congenital lymphedema. Lower limb involvement constitutes most cases of primary lymphedema; however, edema may also involve the upper extremities, face, or genitalia. In this patient the distal part of the extremity is affected initially, with proximal extension occurring later.

Differentially we thought parasitic infestation, infection, deep vein thrombosis, secondary to tumors, nodal resection and trauma. In our case filariasis was excluded by doing CFT for filaria, doppler ultrasonography of affected limbs and abdominal ultrasonography was done and no evidence of deep vein thrombosis and obstructive lesions was found. This patient had no history of trauma and no history of surgical procedure.

Isotopic lymphangioscintigrams is generally considered the gold standard for the diagnosis of lymphedema. The procedure is minimally invasive, repeatable, easy to perform, and harmless to the 
lymphatic endothelium. Lymphangioscintigrams in patients with primary lymphedema display absent or delayed tracer transport, lack or paucity of lymphatic channels, retrograde diffusion (backflow) and poorly visualized or absent regional lymph nodes. Several author found absent or delayed tracer transport in primary lymphedema by isotope lymphangioscintigrphy ${ }^{9}$. Lymphoscintigraphy was done in this baby and non-visualized lymphatic channel and lymph nodes in left lower limb with no ascent of tracer into the lymphatic vessels of the left lower extremity was found. Gates ${ }^{10}$ also reported a case of idiopathic primary congenital lymphedema and he found aplasia of lymphatic channel in isotopic lymphoscintigraphy.

The management of primary lymphedema is usually conservative and successful for most patients. Avoiding prolonged standing, elevation of the affected limb and skin care is sufficient for the uncomplicated mild cases of lymphedema. A particular emphasis on skin care is important to reduce the increased risk of cellulitis and lymphangitis. Maneuvers that enhance lymphatic drainage such as compression (pneumatic pumps and compressive garments), special exercise and manual lymphatic drainage are also recommended ${ }^{11}$. When medical management fails, surgical intervention is indicated as the last option. There are numerous potential surgical procedures, such as resection approach, debulking, buried dermal flaps, microsurgical techniques like creation of anastomoses between lymphatic vessels and veins, between lymph nodes and veins, and between distal and proximal lymphatics, but complications are common and there is only $30 \%$ success rate 1 . Conservative management as well as surgical consultation was taken in our patient and no further deterioration occurred for next 12 months.

\section{Conclusion:}

Idiopathic congenital lymphedema is a rare disease in children. The patients with lymphedema exhibit psychological and physical burden. Diagnosis of congenital lymphedema is made from a carefully taken clinical history including family history and findings in the physical examination. Physical examination findings can be quite helpful in distinguishing lympedema from other causes of swollen extremities. In cases where the diagnosis is unclear, lymphangioscintigrphy should be performed to confirm the presence or absence of lymphatic dysfunction. Management of primary lymphedema is conservative in most cases. For patient who fails to respond to conservative management, surgical intervention may be suggested.

\section{References:}

1. Smeltzer DM, Stickler GB, Schirger A. Primary lymphedema in children and adolescents: a follow-up study and review. Pediatrics. 1985; 76:206-18

2. Wright NB, Carty HM. The swollen leg and primary lymphedema. Arch Dis Child. 1994; 71: 44-49.

3. Fonkalsrud EW, CoulsonWF. Management of congenital lymphedema in infant and children. Ann Surg.1973;177:280-85

4. Shinawi M. Lymphedema of the Lower Extremity:Is It Genetic or Nongenetic? Clinical Pediatrics. 2007; 46(9): 835-41

5. Wananukul S, Jittitaworn S. Primary congenital lymphedema involving limbs and genitalia. J Med Assoc Thai. 2005; 88 (12): 1958-61

6. Evans AL, Brice G, Sotirova V, Mortimer P, Beninson J, Burnand K, et al. Mapping of primary congenital lymphedema to the $5 \mathrm{q} 35.3$ region. $A m$ J Hum Genet. 1999; 64: 547-55

7. Ogata T, Sato S, Hasegawa Y, Kosaki K. Lymph stasis in a boy with Noonan syndrome: implication for the development of skeletal features. Endocr J. 2003; 50: 319-24

8. Sim YJ, Seo JH. Congenital lymphedema of the upper extremity. Eur J Phys Rehabil Med. 2008; 44: 89-91

9. Eric WF, Walter FC. Management of Congenital Lymphedema in Infants and Children. Ann. Surg. 1973; 77(3): 280-85

10. Gates G F, Dore EK. Primary congenital lymphedema in infancy evaluated by isotope lymphoscintigraphy. Journal Of Nuclear Med.1971; 12(6): 315-17

11. Rockson SG. Lymphedema. Am J Med. 2001; 110: 288-95 